\title{
News and Events
}

Sustainable Cities: Sustainable Development (The urban agenda in developing countries 3-4 April 200, at the Oxford Bookes University, Gipsy Lane Campus.

Information: e-mail: rwzetter@brookes.ac.uk

\section{Building Better Communities}

5 April 1000, TCPA, London.

Information: tel: +44 (0)2079308930

\section{Housing Density and Design Quality}

17 May 2000, Royal Town Planning Institute, Yorkshire Branch, York.

Information: Paula Nolan, tel: +44 (0)113 246 5714, fax: +44 (0)113 242 6791, e-mail: yorkshire@cix.co. uk

Financing Transportation for the $21^{\text {st }}$ Century 14-15 June 2000, Intelligent Transport Society for the United Kingdom, Glasgow.

Information: Corinne Paine, tel: +44 (0)1926 614420

CNU 2000: The Politics of Place

$8^{\text {th }}$ Annual Congress for the New Urbanism. 15-18 June 2000, Portland, Oregon, USA.

Information: tel: +1 (800) 7887077

Cities for the Well Being of Children

$27^{\text {th }}$ International Conference on Making Cities Livable

4-8 July 2000, Vienna, Austria.

Information: P.O. Box 7586, Carmel CA 93921, tel: +1 (831) 626 9080, fax: +1 (831) 624 5126, e-mail: suzanne.lennard@livablecities.org

Summer Programme in Architecture and Urban Design 2000

5-11 July, Berlin; 12-18 July, Paris; 19-26 July,
London; 27 July to 4 August, Oxford. £1817, course credits available.

Information: John Stevenson, Course Leader, fax: +44 (0)1865 483298; Maureen Jones, Course administrator, fax: +44 (0)1865 484884

TIA 2000 Third International Conference

Sustainable Buildings for the $21^{\text {st }}$ Century: Teaching Issues, Tools and Methodologies for Sustainability

9-12 July 2000, Somerville College, Oxford, UK. Information: Vivien Walker, School of Architecture, Oxford Brookes University, Gipsy Lane, Headington, Oxford OX3 0BP, fax: +44 (0)1865 483298, e-mail: tia@brookes.ac.uk

Online booking form: http://www.brookes.ac. uk/schools/arch/TIAreg.htm

\section{Planning and Turning Point}

AESOP XIV Congress

18-23 July 2000, Brno, Czech Republic.

Information: tel: +420 (5) 4324 5212, fax: +420 (5)

4321 1134, e-mail: taservis@brn.pvtnet.cz

\section{Better Spaces for Small Towns}

International Federation for Housing \& Planning $6^{\text {th }}$ IFHP Urban Planning and Design Summer School

30 July to 12 August 2000, Helsinki - Porvoo Jyväskylä, Finland.

Information: tel: +358 (9) 451 2147, fax: +358 (9) 4514071

Second Sustainable Cities Network Conference 2-5 November 2000, Manchester, UK.

Information: David Gibbs, tel: +44 (0)1482 465330, fax: +44 (0)1482 466340, e-mail: D.C.Gibbs@geo. hall.ac.uk 